\title{
Alimentation oligo-minérale du Pin maritime (Pinus pinaster Soland in Ait) en relation avec quelques caractéristiques physico-chimiques des sols sableux des Landes de Gascogne
}

\author{
E. Saur \\ Station d'Agronomie, INRA, BP 131, 33140 Pont-de-La-Maye, France
}

(reçu le 30-3-1988, accepté le 26-7-1988)

\begin{abstract}
Résumé - L'objectif de ce travail est de tenter de relier la nutrition minérale (plus particulièrement oligo-minérale) du pin maritime à des troubles de croissance que manifeste ce dernier dans certains peuplements sur sols sableux des Landes de Gascogne. Pour ce faire, on a analysé des échantillons de feuilles, d'écorces et de sols, prélevés dans 5 stations représentatives. Les résultats montrent que l'oligominéralomasse ( $\mathrm{Mn}, \mathrm{Fe}, \mathrm{Zn}, \mathrm{Cu}$ ) des 5 profils mesurés sur une profondeur de 1 mètre se situe à un niveau très faible, en raison de la nature essentiellement quartzeuse de la roche mère et que, par ailleurs, les plus fortes concentrations en éléments-traces sont rencontrées dans les horizons de surface - en relation, sans doute, avec la prédominance du processus de recyclage biologique par la végétation. Dans les 5 stations examinées, l'aluminium se singularise par sa concentration importante, aussi bien dans l'écorce que dans les feuilles, alors que, à l'opposé, les teneurs en cuivre sont très peu élevées (3,5 ppm/MS en moyenne) et diminuent avec l'apport d'engrais phosphaté sur les jeunes plants, ce qui conduit à émettre l'hypothèse d'une possible malnutrition en cet élément de certains peuplements de Pin maritime des Landes de Gascogne.
\end{abstract}

nutrition des plantes - oligo-éléments - analyse de feuille - analyse de sol - Pinus pinaster podzol - Landes de Gascogne

Summary - Relationships between the micronutrient nutrition of Pinus pinaster Soland in Ait and properties of sandy soils in Landes de Gascogne (France). On various sites in the Landes de Gascogne, deformations on the shoots occur in Pinus pinaster, giving rise to abnormal form. In order to determine the cause of the deformation several plant and soil analysis have been made with a particular attention on micro-nutrients : $\mathrm{Mn}, \mathrm{Zn}, \mathrm{Cu}, \mathrm{B}$. In winter 1986-1987, soil, needle and bark samples were taken from five sites representative of three kinds of podzol, with young plants or adults, with and without deformed pines.

The main conclusions of this work are :

1) a low level of soil elements such as manganese, zinc and copper is connected with quartzeous parent material;

2) a prominent part of plants in bioelement mobilization and redistribution on soil;

3) some important soil mineral content in comparison to the need of pines;

4) a general lowness of copper rates in different plant organs $(3,5 \mathrm{ppm} D W$ in leaves) in reference to deficiency rates of other coniferous species, and a decrease of copper rates in leaves with $P$ fertilization.

plant nutrition - micro-nutrient - foliar analysis - soil analysis - Pinus pinaster - podzol Landes de Gascogne 


\section{Introduction}

Le sable des Landes, de nature essentiellement quartzeuse, présente de faibles réserves en éléments minéraux et notamment en oligo-éléments. Cette pauvreté originelle peut être à l'origine de phénomènes de malnutrition, comme par exemple la carence en cuivre sur maïs, décrite dès 1954 par Redlich, carence observée principalement dans les secteurs où la forte hydromorphie conduit à la formation de sols de nature quasi tourbeuse.

Compte tenu de ce contexte pédologique très particulier, il nous a paru intéressant de tenter une approche de la connaissance de l'alimentation oligo-minérale du Pin maritime, essence forestière dominante en Aquitaine où elle couvre près d'un million d'hectares. Une alimentation oligo-minérale défectueuse constitue en effet l'une des hypothèses avancées pour expliquer certains troubles de croissance qui se manifestent localement sur arbres adultes (conformation flexueuse des axes principaux et des jeunes pousses) ou sur jeunes semis (flexuosité des pousses de $3^{\circ}$ année, verse) - et ce, semble-t-il, indépendamment d'attaques parasitaires identifiables.

Ces malformations se rencontrent dans tous les types de terrains, mais beaucoup plus rarement cependant dans les sols dunaires; les accidents sont surtout fréquents dans les peuplements fertilisés en phosphore, bien ou très bien "venant", où les techniques de la sylviculture moderne favorisent la croissance.

La nature des troubles observés pourrait conduire à incriminer une alimentation insuffisante en cuivre : d'une part, en raison du rôle que joue ce métal dans les processus de lignification (Rahimi et Bussler, 1974) et, d'autre part, en raison de la similitude de ces troubles avec ceux traduisant des carences en cuivre reconnues chez le Douglas (Van Goor, 1963; Van Goor, 1965; Strullu et Bonneau, 1978), le mélèze (Penningsfeld, 1964) et le Pin radiata (Ruiter, 1969; Will, 1972; Turvey, 1984).

Le but de cette note est d'estimer les teneurs en oligo-éléments des aiguilles du Pin maritime et des sols dans différents types de stations des Landes et de voir si des carences en oligo-éléments et notamment en cuivre sont plausibles.

La qualité de l'alimentation a été définie à partir des résultats fournis par l'analyse du végétal (feuilles et écorces), qui ont été rapprochés des principales caractéristiques chimiques de profils pédologiques des stations où se situent les peuplements examinés.

\section{Matériels et Méthodes}

\section{Présentation des stations}

Des échantillons de sols et de végétaux ont été prélevés dans 5 stations qui se répartissent comme suit :

\section{Lande humide (Berganton)}

Sol de type podzol humique à Molinie; peuplement sain de 24 ans fertilisé; traitements :

- $T$ : témoin,

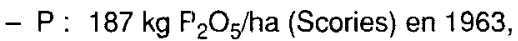
$60 \mathrm{~kg} \mathrm{P} 2 \mathrm{O}_{5} /$ ha (Phosphates naturels) en 1974 .

\section{Lande sèche (Sore)}

Sol de type podzol humo-ferrugineux ou podzol humique à $\mathrm{A} 2$ et alios;

peuplement sain de 25 ans fertilisé à la plantation (120 unités $\mathrm{P}_{2} \mathrm{O}_{5}$ par hectare cadastral). 


\section{Dune littorale (Le Porge)}

Sol de type lette maigre sur sable dunaire; peuplement sain de 29 ans fertilisé à la plantation (120 unités $\mathrm{P}_{2} \mathrm{O}_{5}$ par hectare cadastral).

\section{Peuplement adulte «mal-venant»} (Saint-Jean-d'lliac)

Lande humide;

sol de type podzol humique hydromorphe à faible accumulation;

peuplement de 16 ans fertilisé à la plantation présentant des déformations des tiges jeunes et âgées.

\section{Peuplement juvénile (Castillonville, Pierroton)}

Lande humide;

sol de type podzol humique hydromorphe à faible accumulation et pseudogley profond; peuplement sain de 3 ans;

traitements :

$-T$ : témoin,

- P1 : $60 \mathrm{~kg} \mathrm{P}_{2} \mathrm{O}_{5} /$ ha (supertriple $45 \%$ ),

-P2 : $120 \mathrm{~kg} \mathrm{P}_{2} \mathrm{O}_{5} /$ ha (supertriple $45 \%$ ).

\section{Modalités de prélèvement des échantillons}

Les échantillons (sols et végétaux) ont été prélevés en février 1987 dans l'ensemble des stations. Cette période n'est pas celle choisie comme standard par les forestiers mais permet néanmoins une étude comparative interstationnelle. Des prélèvements ont également été effectués en décembre 1986. Les résultats analytiques différant assez peu de ceux de février 1987 , on pourra envisager de comparer assez valablement l'ensemble des résultats de février 1987 aux normes de composition foliaire dont on dispose et aux chiffres relevés dans la bibliographie.

\section{Prélèvement des échantillons végétaux}

L'échantillonnage a porté sur les aiguilles de première et de deuxième années du troisième verticille détachées à l'aide d'une carabine à plombs, ainsi que sur l'écorce prélevée à $1,20 \mathrm{~m}$ du sol. Nous avons choisi 10 arbres représentatifs du peuplement par leur taille. Un seul échantillon composite par type d'organe et par station est obtenu par le mélange des 10 prélèvements élémentaires correspondant aux 10 arbres échantillonnés.

\section{Prélèvement des échantillons de sol}

Dans chaque station, nous avons creusé une fosse pédologique permettant la description des horizons. Pour chaque horizon, nous avons effectué un prélèvement de sol destiné aux analyses chimiques et un prélèvement d'un volume connu $(257,8 \mathrm{ml})$ obtenu par enfoncement horizontal d'un cylindre métallique de $7 \mathrm{~cm}$ de diamètre, destiné à la mesure de la densité apparente. Dans le cas de la litière, nous avons substitué à la détermination de la densité apparente celle de la masse surfacique (prélèvement de $1 \mathrm{~m}^{2}$ de litière).

\section{Déterminations réalisées}

\section{Analyse des échantillons végétaux}

Après séchage à l'étuve à $105^{\circ} \mathrm{C}$, les échantillons ont été broyés et minéralisés par voie sèche (calcination de 4 heures à $450^{\circ} \mathrm{C}$ ). Les cendres ont été reprises à chaud par $5 \mathrm{ml}$ de $\mathrm{HCl} 6 \mathrm{~N}$ puis ajustées à $100 \mathrm{ml}$ avec de l'eau distillée. $\mathrm{K}, \mathrm{Ca}, \mathrm{Mg}, \mathrm{Na}, \mathrm{Cu}, \mathrm{Mn}, \mathrm{Zn}, \mathrm{Fe}$ ont été dosés par absorption atomique de flamme. L'aluminium a été dosé par colorimétrie du complexe Al-ériochromocyanine (Juste, 1965). Après minéralisation par calcination à $550^{\circ} \mathrm{C}$ de $5 \mathrm{~g}$ de poudre, puis reprise dans $5 \mathrm{ml}$ d'acide acétique à $25 \%$, le bore a été dosé par une colorimétrie à l'auto-analyseur, faisant intervenir la réaction entre l'azométhine $\mathrm{H}$ et le bore en milieu faiblement acide (Rodier, 1984).

L'azote organique a été dosé à l'auto-analyseur par colorimétrie du complexe d'un sel de diazonium, après minéralisation par voie humide $\left(5 \mathrm{ml} \mathrm{H}_{2} \mathrm{SO}_{4}\right.$ concentré $+15 \mathrm{ml} \mathrm{H}_{2} \mathrm{O}_{2} 110 \mathrm{~V}$ $\left.+3 \mathrm{~g} \mathrm{~K}_{2} \mathrm{SO}_{4}+3 \mathrm{mg} \mathrm{Se}\right)$ de $0,2 \mathrm{~g}$ de poudre végétale.

\section{Evaluation de la minéralomasse foliaire}

Elle a été obtenue, pour chaque élément analysé, en faisant le produit de la concentration de 
ce dernier par la biomasse foliaire exprimée en poids de matière sèche de feuilles par hectare. On a tenu compte pour ce faire de valeurs de biomasse effectivement mesurées en 1979 dans la même parcelle (Traitement $P$ de Berganton) par Lemoine ot al. (1986). II convient donc de bien préciser que la valeur de minéralomasse ainsi obtenue ne représente qu'une estimation approchée de la minéralomasse réelle actuelle.

\section{Analyse des échantillons de sol}

Nous avons pesé les échantillons de sol destinés au calcul des densités apparentes après passage à l'étuve à $105^{\circ} \mathrm{C} . \mathrm{Mn}, \mathrm{Fe}, \mathrm{Zn}$ et $\mathrm{Cu}$ ont été dosés en absorption atomique de flamme après attaque de $1 \mathrm{~g}$ de sol par $10 \mathrm{ml}$ d'eau régale $\left(7,5 \mathrm{ml} \mathrm{HCl}+2,5 \mathrm{ml} \mathrm{H} \mathrm{NO}_{3}\right)$ à ébullition pendant $2 \mathrm{~h}$. Pour chacun de ces éléments, les valeurs mesurées peuvent donc être assimilées aux teneurs totales. $\mathrm{K}, \mathrm{Ca}, \mathrm{Mg}$ échangeables ont été dosés en absorption atomique de flamme, après extraction à l'acétate d'ammonium en solution normale. L'azote a été dosé selon la méthode Kjeldahl. La teneur en matière organique a été évaluée par la mesure de la perte de masse d'un échantillon porté à $800^{\circ} \mathrm{C}$ pendant $6 \mathrm{~h}$ (perte au feu).

\section{Evaluation de la minéralomasse des pro-} fils

Les minéralomasses ont été calculées pour chaque horizon à partir de son épaisseur, de sa densité apparente et de la teneur en chaque élément. La minéralomasse du profil a été obtenue par sommation, sur une profondeur de $1 \mathrm{~m}$, des minéralomasses des horizons successifs du profil.

\section{Résultats obtenus et discussion}

Composition minérale des échantillons
végétaux

Quelle que soit la station considérée, les mêmes tendances se dégagent en matière d'évolution avec l'âge des concentrations dans les feuilles (Tableau I) : c'est ainsi que le calcium, l'aluminium, le fer et le manganèse s'accumulent plus intensément dans les feuilles de $2^{\mathrm{e}}$ année alors que, à l'opposé, les concentrations de tous les autres éléments ont tendance à diminuer avec l'âge. Ce phénomène avait déjà été signalé par Demounem (1979) pour ce qui concerne l'azote.

L'écorce présente des teneurs très faibles en éléments majeurs ( $N, P, K, M g$ ) et relativement faibles pour certains oligoéléments ( $\mathrm{Mn}, \mathrm{Zn}, \mathrm{Cu}, \mathrm{B})$. Au contraire, l'aluminium et le fer semblent s'accumuler dans ce tissu mort.

Tableau I. Moyennes des teneurs en éléments minéraux dans 3 organes de Pinus pinaster calculées pour les 4 peuplements adultes.

\begin{tabular}{|c|c|c|c|c|c|c|c|c|c|c|c|}
\hline & $\begin{array}{l}N \\
\%\end{array}$ & $\begin{array}{l}P \\
\%\end{array}$ & $\begin{array}{l}K \\
\%\end{array}$ & $\begin{array}{l}\mathrm{Ca} \\
\%\end{array}$ & $\begin{array}{l}M g \\
\%\end{array}$ & $\begin{array}{l}M n \\
p p m\end{array}$ & $\begin{array}{l}F e \\
p p m\end{array}$ & $\begin{array}{l}Z n \\
p p m\end{array}$ & $\begin{array}{l}\text { Cu } \\
\text { ppm }\end{array}$ & $\begin{array}{l}\text { B } \\
\text { ppm }\end{array}$ & $\begin{array}{l}A l \\
p p m\end{array}$ \\
\hline \multicolumn{12}{|c|}{ Aiguilles, $1^{50}$ année, $3^{\circ}$ verticille : } \\
\hline Moyenne & 1,01 & 0,09 & 0,45 & 0,17 & 0,13 & 80,4 & 51,8 & 36,0 & 3,4 & 15,9 & 215 \\
\hline Ecart type & 0,18 & 0,01 & 0,08 & 0,01 & 0,02 & 62 & 7,9 & 2,9 & 0,4 & 2,9 & 62 \\
\hline \multicolumn{12}{|c|}{ Aiguilles, $2^{e}$ année, $3^{e}$ verticille : } \\
\hline Moyenne & 0,91 & 0,07 & 0,33 & 0,29 & 0,12 & 131 & 66,4 & 31,4 & 2,4 & 13,6 & 240 \\
\hline Ecart type & 0,17 & 0,02 & 0,02 & 0,06 & 0,02 & 151 & 11,8 & 8,0 & 0,6 & 3,2 & 78 \\
\hline \multicolumn{12}{|c|}{ Ecorce du tronc à $1,20 \mathrm{~m}$ : } \\
\hline Moyenne & 0,15 & 0,05 & 0,01 & 0,17 & 0,02 & 11,9 & 84,2 & 14,7 & 2,2 & 4,7 & 365 \\
\hline Ecart type & 0,02 & 0,003 & 0,003 & 0,03 & 0,007 & 5,9 & 49,2 & 7,3 & 0,5 & 0,5 & 89 \\
\hline
\end{tabular}


Les résultats d'analyse, aussi bien des feuilles que de l'écorce, confirment l'abondance notable de l'aluminium dans ces organes (plus de 400 ppm dans les écorces provenant des stations du Porge et de Sore et plus de 200 ppm dans les aiguilles). Dans une certaine mesure, le Pin maritime peut donc être considéré comme une espèce accumulant l'aluminium - ce qui est en accord avec les observations déjà faites par Juste (1965) et par Humphreys, qui avait signalé en 1964 l'accumulation de ce métal dans des résineux se développant sur des sols acides de l'Est australien : $745 \mathrm{ppm}$ chez Pinus radiata et 1293 ppm chez Pinus taeda. Zöttl (1985) a mesuré des teneurs en aluminium de 120 à $130 \mathrm{ppm}$ dans les feuilles d'épicéas âgés d'un an. Plus récemment, Messenger (1975) a confirmé le rôle important joué par les résineux sur le processus de podzolisation précisément en raison de la grande richesse en aluminium de leurs retombées foliaires.

Le Tableau II fait bien apparaître que, dans les peuplements adultes, les concentrations en éléments varient d'une station à l'autre. Les teneurs en azote sont minimales à Sore et au Porge, traduisant une carence azotée en lande sèche et sable dunaire. A l'inverse, les teneurs en phosphore sont élevées à Sore et au Porge et faibles à Berganton et SaintJean-d'lllac, reflétant ainsi l'antagonisme
N/P. Les concentrations en fer (52 ppm en moyenne) sont faibles en comparaison des seuils de carence de 30-40 ppm déterminés par Woods (1983) pour Pinus radiata et de $104 \mathrm{ppm}$ pour les jeunes plants de Picea sitchensis (Gessel et al., 1979). Les teneurs en potassium sont maximales à Berganton et minimales à Saint-Jean-d'lllac et au Porge. Le rapport $\mathrm{N} / \mathrm{K}$, plus élevé à Berganton qu'à SaintJean-d'lllac, pourrait être en relation avec les problèmes de qualité du bois, déjà constatés chez le Pin maritime.

$\mathrm{La}$ nutrition en $\mathrm{Ca}, \mathrm{Mn}, \mathrm{Zn}$ et $\mathrm{B}$ est convenable pour l'ensemble des stations. Le manganèse atteint des concentrations plus élevées pour la station du Porge, ce qui reflète la plus grande richesse du profil en cet élément (Tableau V). La comparaison entre Saint-Jean-d'lllac (peuplement malvenant de lande humide) et Berganton (peuplement sain de lande humide) n'apporte pas d'élément de réponse définitif concernant la cause nutritionnelle des déformations de rameaux d'arbres adultes.

Le Tableau III met également en évidence une accumulation plus importante de la plupart des éléments ( $N, \mathrm{Ca}, \mathrm{Mn}, \mathrm{Fe}$, $\mathrm{Zn}, \mathrm{Cu})$ dans les aiguilles de première année du semis de 3 ans (Castillonville) que dans celles provenant des peuplements adultes (Tableau II). Cette observation est sans doute à mettre en relation avec la colonisation des horizons de sur-

Tableau II. Teneurs en éléments minéraux des aiguilles de $1^{1 \mathrm{e}}$ année ( $3^{\circ}$ verticille) de Pinus Pinaster adulte.

\begin{tabular}{lccccccccccc}
\hline Station & $\begin{array}{l}N \\
\%\end{array}$ & $\begin{array}{l}P \\
\%\end{array}$ & $\begin{array}{l}K \\
\%\end{array}$ & $\begin{array}{l}C a \\
\%\end{array}$ & $\begin{array}{l}M g \\
\%\end{array}$ & $\begin{array}{l}M n \\
p p m\end{array}$ & $\begin{array}{l}\text { Fe } \\
p p m\end{array}$ & $\begin{array}{l}Z n \\
p p m\end{array}$ & $\begin{array}{l}\text { Cu } \\
\text { ppm }\end{array}$ & $\begin{array}{l}\text { B } \\
p p m\end{array}$ & $\begin{array}{l}A l \\
p p m\end{array}$ \\
\hline Saint-Jean-d'llac & 1,20 & 0,09 & 0,39 & 0,19 & 0,15 & 51,6 & 58,2 & 33,8 & 3,8 & 19,2 & 251 \\
Le Porge & 0,92 & 0,11 & 0,48 & 0,18 & 0,15 & 173 & 56,8 & 39,5 & 3,8 & 12,9 & 283 \\
Sore & 0,81 & 0,10 & 0,39 & 0,17 & 0,12 & 43,4 & 51,7 & 37,2 & 3,1 & 14,1 & 177 \\
Berganton T & 1,13 & 0,07 & 0,55 & 0,16 & 0,11 & 52,8 & 40,6 & 33,5 & 3,0 & 17,6 & 150 \\
Berganton P & 1,03 & 0,08 & 0,52 & 0,16 & 0,12 & 75,0 & 50,4 & 34,2 & 2,8 & 18,4 & 131 \\
\hline
\end{tabular}


Tableau III. Teneur en éléments minéraux des aiguilles de $1^{\text {re }}$ année de jeunes plants de Pinus Pinaster.

\begin{tabular}{lccccccccccc}
\hline & $\begin{array}{l}N \\
\%\end{array}$ & $\begin{array}{l}P \\
\%\end{array}$ & $\begin{array}{l}K \\
\%\end{array}$ & $\begin{array}{l}C a \\
\%\end{array}$ & $\begin{array}{l}M g \\
\%\end{array}$ & $\begin{array}{l}M n \\
p p m\end{array}$ & $\begin{array}{l}F e \\
p p m\end{array}$ & $\begin{array}{l}Z n \\
p p m\end{array}$ & $\begin{array}{l}C u \\
p p m\end{array}$ & $\begin{array}{l}B \\
p p m\end{array}$ & $\begin{array}{l}A l \\
p p m\end{array}$ \\
\hline Témoin & 2,35 & 0,11 & 0,45 & 0,25 & 0,10 & 266 & 67,6 & 57,6 & 5,0 & 15,4 & 209 \\
Traitement P1 & 1,97 & 0,14 & 0,56 & 0,23 & 0,10 & 232 & 44,2 & 44,2 & 4,0 & 14,1 & - \\
Traitement P2 & 1,74 & 0,15 & 0,53 & 0,27 & 0,12 & 171 & 47,1 & 38,1 & 2,0 & 14,9 & - \\
\hline
\end{tabular}

face, plus riches en éléments nutritifs, par le système racinaire du jeune semis, ainsi qu'avec le caractère plus exigeant en éléments nutritifs des jeunes plants. Cette accumulation plus intense des éléments minéraux par le jeune semis a pour corollaire une plus grande richesse en cendres des tissus de ce dernier $(1,9 \%$ en moyenne pour les arbres adultes du site de Berganton, à proximité de celui de Castillonville où la teneur moyenne en cendres des tissus est de l'ordre de 2,3\%).

$\mathrm{Ce}$ qui retiendra particulièrement notre attention dans le Tableau III est l'effet des doses croissantes de phosphate sur la composition minérale des aiguilles. Nous constatons une chute des concentrations en cuivre (de 5 à $2 \mathrm{ppm}$ ) lors de l'apport des doses $\mathrm{P} 1$ et $\mathrm{P} 2$ de phosphate, alors que les teneurs des autres éléments restent pratiquement inchangées (à l'exception de $\mathrm{N}$ et $\mathrm{Zn}$ ). Le cuivre se comporterait dans cet essai comme un facteur limitant secondaire, le facteur primaire étant le phosphore qui augmente notablement la croissance des arbres jeunes et adultes (Bonneau et al., 1972).

Le résultat le plus important à souligner, à la suite de cette campagne systématique d'analyse du matériel végétal, est le très faible niveau des teneurs en cuivre, toujours inférieures à $5 \mathrm{ppm}$ et oscillant, dans la majorité des cas, autour d'une valeur moyenne de $3,5 \mathrm{ppm}$. Or, selon Strullu et Bonneau (1978), le seuil de carence est de $3 \mathrm{ppm}$ pour l'Epicéa de Sitka et, selon Woods (1983), de 2 à 3 ppm pour le Pin radiata. Les seules teneurs en cuivre publiées sur le Pin maritime sont celles fournies par Bonneau et al. (1968) : elles varient entre 3,1 et $9,6 \mathrm{ppm}$ pour différentes origines portugaises et entre 5,0 et $8,4 \mathrm{ppm}$ pour les origines landaises. On notera que, chez les plantes annuelles de grande culture, la valeur de 4 à $5 \mathrm{ppm}$ dans les parties végétatives est également considérée comme valeur critique dénotant une alimentation cuprique insuffisante (Coppenet, 1968; Loue, 1986). Ce seuil est

Tableau IV. Minéralomasses foliaires exprimées en $\mathrm{kg} / \mathrm{ha}$ sur le site de Berganton (traitement P) pour une biomasse foliaire de 7,4 tha* .

\begin{tabular}{llllllllllll}
\hline & $N$ & $P$ & $K$ & $\mathrm{Ca}$ & $\mathrm{Mg}$ & $\mathrm{Mn}$ & $\mathrm{Fe}$ & $\mathrm{Zn}$ & $\mathrm{Cu}$ & $\mathrm{B}$ & $\mathrm{Al}$ \\
\hline $\begin{array}{l}\text { Valeurs mesurées } \\
\text { en 1979 (Lemoine } \\
\quad \text { et al. à paraître) }\end{array}$ & 65 & 7 & 29 & 21 & 9 & - & - & - & - & - & - \\
$\begin{array}{l}\text { Valeurs estimées } \\
\text { en 1987 }\end{array}$ & 77 & 6 & 38 & 12 & 9 & 0,6 & 0,4 & 0,3 & 0,02 & 0,1 & 1 \\
\hline
\end{tabular}

* : Selon Lemoine et al. (1986). 
d'ailleurs celui qui est pris en compte pour le diagnostic de la carence en cuivre chez le maïs cultivé dans les sols de défriche récente des Landes de Gascogne.

La connaissance des teneurs minérales des aiguilles du peuplement de Berganton (traitement $P$ ) nous a permis de calculer les minéralomasses foliaires estimées (Tableau IV) qui ont l'intérêt de donner une idée des besoins réels d'un peuplement de Pin maritime et qui représentent des résultats inédits en ce qui concerne les oligo-éléments.

Caractéristiques chimiques et minéralomasses des sols

Les minéralomasses des 5 profils, évaluées sur une profondeur totale de $1 \mathrm{~m}$ pour un certain nombre de composantes du sol, sont indiquées dans le Tableau V. Ces chiffres permettent de comparer les réserves minérales de chaque station mais ne préjugent en rien des quantités réellement disponibles pour le végétal; en effet, ils ne tiennent compte ni de la colonisation des niveaux plus profonds par les racines ni de la biodisponibilité effective des éléments, notamment pour ce qui concerne l'azote et les éléments en traces dont la fraction totale a été évaluée.

Les résultats de l'analyse détaillée des 5 profils sont rassemblés dans les Tableaux $V I$ à $X$. Ces tableaux font apparaître, ce qui était assez prévisible, que la roche mère des 5 stations, constituée de sables quaternaires, a une composition moyenne variant peu d'une station à l'autre. Elle est très faiblement pourvue en éléments métalliques; ceci est particulièrement vrai pour le cuivre, dont la concentration totale oscille selon les stations de 0,6 à 1,3 ppm.

On observe pour la plupart des éléments une diminution sensible des concentrations avec la profondeur, ce qui signifie que, compte tenu de la pauvreté de la roche mère dans la plupart d'entre

Tableau V. Propriétés physico-chimiques des sols des 5 stations, calculées sur $1 \mathrm{~m}$ de profondeur.

\begin{tabular}{|c|c|c|c|c|c|c|c|c|c|c|c|}
\hline \multirow[b]{2}{*}{ Station } & \multirow{2}{*}{$\begin{array}{l}\text { Mat. } \\
\text { org. } \\
\%\end{array}$} & \multicolumn{2}{|c|}{ pH-eau } & \multirow{2}{*}{$\begin{array}{l}N \\
\text { Kjeld. } \\
\text { tha }\end{array}$} & \multicolumn{3}{|c|}{ El. échang. (acét. $\mathrm{NH}_{4}$ ) } & \multicolumn{4}{|c|}{ Eléments totaux (eau régale) } \\
\hline & & & $\max$ & & $\begin{array}{l}K \\
\mathrm{~kg} / \mathrm{ha}\end{array}$ & $\begin{array}{l}\text { Ca } \\
\text { T/ha }\end{array}$ & $\begin{array}{l}\mathrm{Mg} \\
\mathrm{kg} / \mathrm{ha}\end{array}$ & $\begin{array}{l}\mathrm{Mn} \\
\mathrm{kg} / \mathrm{ha}\end{array}$ & $\begin{array}{l}\text { Fe } \\
\text { t/ha }\end{array}$ & $\begin{array}{l}\mathrm{Zn} \\
\mathrm{kg} / \mathrm{ha}\end{array}$ & $\begin{array}{l}\mathrm{Cu} \\
\mathrm{kg} / \mathrm{ha}\end{array}$ \\
\hline & & $3, \mathrm{c}$ & 4,7 & & & 0,8 & 165 & 140 & 6,76 & 40,9 & 14,4 \\
\hline Le Porge & 0,3 & 4,4 & 5, & 1,14 & 19 & 1,3 & 24 & 309 & 13,7 & 84,9 & 15,7 \\
\hline Sore & 2,3 & 3,8 & 5,0 & 4,65 & 223 & 1,75 & 272 & 213 & 36,84 & 71,6 & 24,4 \\
\hline & 1,2 & 3,5 & 5,0 & - & 108 & 0 & & 167 & 7,22 & 31,3 & 31,0 \\
\hline Castillonville & 2,4 & 3,7 & 5,2 & 10,4 & 202 & 1,58 & 744 & 126 & 7,79 & 64,8 & 23,4 \\
\hline
\end{tabular}

Tableau VI. Analyses de sols, Site de Saint-Jean-d'illac.

\begin{tabular}{|c|c|c|c|c|c|c|c|c|c|c|c|c|c|}
\hline Horizon & $\begin{array}{l}\text { Profon- } \\
\text { deur } \\
(\mathrm{cm})\end{array}$ & $\begin{array}{l}\text { Den- } \\
\text { sité } \\
\text { app. }\end{array}$ & $\begin{array}{l}\mathrm{pH}- \\
\text { eau }\end{array}$ & $\begin{array}{l}\mathrm{pH}- \\
\mathrm{KCl}\end{array}$ & $\begin{array}{l}N \\
\text { Kjeld. } \\
\text { p. } 1000\end{array}$ & $\begin{array}{l}\text { El. éc } \\
K \\
p p m\end{array}$ & $\begin{array}{c}\text { chang. (ac } \\
\mathrm{Ca} \\
\mathrm{ppm}\end{array}$ & $\begin{array}{c}\left.\mathrm{NH}_{4}\right) \\
\mathrm{Mg} \\
\mathrm{ppm}\end{array}$ & $\begin{array}{l}\text { Elém } \\
\text { Mn } \\
\text { ppm }\end{array}$ & $\begin{array}{l}\text { nts totau } \\
F e \\
p p m\end{array}$ & $\begin{array}{l}x \text { (eau } \\
Z n \\
p p m\end{array}$ & $\begin{array}{l}\text { égale) } \\
\text { Cu } \\
\text { ppm }\end{array}$ & $\begin{array}{l}\text { Mat. } \\
\text { org. } \\
\%\end{array}$ \\
\hline & & 0,03 & 4,3 & & & 395 & 384 & 86 & 22 & 097 & 66 & 8,6 & 70 \\
\hline & & & & & & 28,5 & 17 & & & 954 & 4,3 & & 9 \\
\hline & & & & & & 36 & & 26 & & & & & 6,7 \\
\hline & & 0,8 & 4,1 & 4, & 4,28 & 26,0 & & 17, & & 823 & 2,6 & 2,3 & 14,8 \\
\hline$B_{2} S$ & & 1,6 & 4,4 & 4 & 0,2 & 5 & 60 & 8 & 13 & 462 & 2,3 & 1,0 & 1,1 \\
\hline & -105 & 1,5 & 4,7 & 5,2 & 0,07 & 4,4 & 48,0 & 7,2 & 10,6 & 375 & 2,3 & 0,7 & 0,3 \\
\hline
\end{tabular}


Tableau VII. Analyses de sols, Site du Porge.

\begin{tabular}{|c|c|c|c|c|c|c|c|c|c|c|c|c|c|}
\hline \multirow[b]{2}{*}{ Horizon } & \multirow{2}{*}{$\begin{array}{l}\text { Profon- } \\
\text { deur } \\
(\mathrm{cm})\end{array}$} & \multirow{2}{*}{$\begin{array}{l}\text { Den- } \\
\text { sité } \\
\text { app. }\end{array}$} & \multirow{2}{*}{$\begin{array}{l}\text { pH- } \\
\text { eau }\end{array}$} & \multirow{2}{*}{$\begin{array}{l}\mathrm{pH}- \\
\mathrm{KCl}\end{array}$} & \multirow{2}{*}{$\begin{array}{l}N \\
\text { Kjeld. } \\
\text { p. } 1000\end{array}$} & \multicolumn{3}{|c|}{ El. échang. (ac. $\left.\mathrm{NH}_{4}\right)$} & \multicolumn{4}{|c|}{ Eléments totaux (eau régale) } & \multirow{2}{*}{$\begin{array}{l}\text { Mat. } \\
\text { org. } \\
\%\end{array}$} \\
\hline & & & & & & $\begin{array}{l}K \\
\mathrm{ppm}\end{array}$ & $\begin{array}{l}\text { Ca } \\
\text { ppm }\end{array}$ & $\begin{array}{l}M g \\
p p m\end{array}$ & $\begin{array}{l}\text { Mn } \\
\text { ppm }\end{array}$ & $\begin{array}{l}F e \\
p p m\end{array}$ & $\begin{array}{l}Z n \\
p p m\end{array}$ & $\begin{array}{l}\mathrm{Cu} \\
\mathrm{ppm}\end{array}$ & \\
\hline L & $-3-$ & 0,03 & 4,2 & 3,8 & 7,76 & 738 & 3053 & 818 & 648 & 602 & 52,2 & 7,5 & 85,3 \\
\hline F.H. & & 1,2 & 4,4 & 3,6 & 0,13 & 86,3 & 473 & 91,4 & 43,1 & 781 & 11,5 & 3,0 & 5,6 \\
\hline$A_{1}$ & 7- 16 & 1,5 & 4,4 & 3,6 & 0,19 & 13,6 & 97,0 & 20,3 & 13,3 & 708 & 4,7 & 1,3 & 0,9 \\
\hline $\mathrm{Bh}$ & $16-43$ & 1,6 & 4,6 & 4,3 & 0,07 & 7,4 & 53,8 & 9,2 & 12,1 & 826 & 3,6 & 0,7 & 0,2 \\
\hline$B_{12}$ & 43- 76 & 1,7 & 4,9 & 4,6 & 0,04 & 5,6 & 51,2 & 8,4 & 18,8 & 742 & 4,0 & 0,7 & 0,2 \\
\hline & $76-97$ & 1,7 & 5,0 & 4,8 & 0,03 & 6,2 & 50,0 & 8,7 & 15,5 & 785 & 5,9 & 0,7 & 0,1 \\
\hline $\mathrm{C}_{1} \mathrm{C}_{2}$ & 96- 98 & & 4,9 & 4,4 & 0,03 & 6,2 & 48,8 & 9,3 & 21,2 & 902 & 4,0 & 0,7 & 0,2 \\
\hline $\mathrm{C}_{2}$ & $98-147$ & 1,5 & 5,2 & 4,9 & 0,03 & 6,8 & 55,4 & 10,1 & 21,0 & 740 & 9,0 & 0,7 & 0,2 \\
\hline
\end{tabular}

Tableau Vill. Analyses de sols, Site de Sore.

\begin{tabular}{|c|c|c|c|c|c|c|c|c|c|c|c|c|c|}
\hline \multirow[b]{2}{*}{ Horizon } & \multirow{2}{*}{$\begin{array}{l}\text { Profon- } \\
\text { deur } \\
\text { (cm) }\end{array}$} & \multirow{2}{*}{$\begin{array}{l}\text { Den- } \\
\text { sité } \\
\text { app. }\end{array}$} & \multirow{2}{*}{$\begin{array}{l}\mathrm{pH}- \\
\text { eau }\end{array}$} & \multirow{2}{*}{$\begin{array}{l}\mathrm{pH}- \\
\mathrm{KCl}\end{array}$} & \multirow{2}{*}{$\begin{array}{l}N \\
\text { Kjeld. } \\
\text { p. } 1000\end{array}$} & \multicolumn{3}{|c|}{ El. échang. (ac. $\left.\mathrm{NH}_{4}\right)$} & \multicolumn{4}{|c|}{ Eléments totaux (eau régaie) } & \multirow{2}{*}{$\begin{array}{l}\text { Mat. } \\
\text { org. } \\
\%\end{array}$} \\
\hline & & & & & & $\begin{array}{l}K \\
\mathrm{ppm}\end{array}$ & $\begin{array}{l}\mathrm{Ca} \\
\mathrm{ppm}\end{array}$ & $\begin{array}{l}M g \\
p p m\end{array}$ & $\begin{array}{l}\mathrm{Mn} \\
\mathrm{ppm}\end{array}$ & $\begin{array}{l}\mathrm{Fe} \\
\mathrm{ppm}\end{array}$ & $\begin{array}{l}Z n \\
p p m\end{array}$ & $\begin{array}{l}\mathrm{Cu} \\
\mathrm{ppm}\end{array}$ & \\
\hline$A_{0}$ & $-4-0$ & 0,03 & 4,0 & 3,6 & 3,96 & 255 & 1286 & 2485 & 76,2 & 678 & 39,9 & 6,3 & 26.5 \\
\hline$A_{1}$ & $0-25$ & 1,3 & 3,8 & 3,0 & 0,33 & 24,8 & 187 & 32,7 & 11,3 & 375 & 6,3 & 1,6 & 3,3 \\
\hline$A_{1}-A_{2}$ & 25- 41 & 1,6 & 4,2 & 3,2 & 0,21 & 11,2 & 87,6 & 13,5 & 9,6 & 348 & 2,6 & 1,3 & 1,4 \\
\hline & $41-63$ & 1,7 & 4,3 & 3,7 & 0,12 & 8,0 & 51,9 & 8,2 & 9,0 & 444 & 2,6 & 0,9 & 0,4 \\
\hline B $50 \%$ & $63-123$ & $1,3^{*}$ & 4,7 & 4,3 & 0,54 & 8,7 & 75,3 & 8,3 & 13,9 & 6681 & 4,5 & 1,9 & 3,5 \\
\hline B $30 \%$ & $63-123$ & $1,3^{*}$ & 4,9 & 4,7 & 0,30 & 5,6 & 116 & 7,2 & 17,6 & 9148 & 4,3 & 1,3 & 2,0 \\
\hline В $20 \%$ & $63-123$ & $1,3^{*}$ & 4,5 & 4,0 & 0,15 & 8,6 & 74,8 & 12,1 & 7,8 & 576 & 2,6 & 1,0 & 0,7 \\
\hline $\mathrm{C}$ & $123-160$ & $1,5^{\star}$ & 5,0 & 4,7 & 0,07 & 6,9 & 61,8 & 9,9 & 8,1 & 777 & 3,4 & 0,6 & 0,5 \\
\hline
\end{tabular}

* : Selon Righi (1977).

Tableau IX. Analyses de sol, Site de Berganton.

\begin{tabular}{|c|c|c|c|c|c|c|c|c|c|c|c|c|c|}
\hline \multirow[b]{2}{*}{ Horizon } & \multirow{2}{*}{$\begin{array}{l}\text { Profon- } \\
\text { deur } \\
\text { (cm) }\end{array}$} & \multirow{2}{*}{$\begin{array}{l}\text { Den- } \\
\text { sité } \\
\text { app. }\end{array}$} & \multirow{2}{*}{$\begin{array}{l}\mathrm{pH}- \\
e a u\end{array}$} & \multirow{2}{*}{$\begin{array}{l}\mathrm{pH}- \\
\mathrm{KCl}\end{array}$} & \multirow{2}{*}{$\begin{array}{l}N \\
\text { Kjeld. } \\
\text { p. } 1000\end{array}$} & \multicolumn{3}{|c|}{ El. échang. (ac. $\mathrm{NH}_{4}$ ) } & \multicolumn{4}{|c|}{ Eléments totaux (eau régale) } & \multirow{2}{*}{$\begin{array}{l}\text { Mat. } \\
\text { org. } \\
\%\end{array}$} \\
\hline & & & & & & $\begin{array}{l}K \\
p p m\end{array}$ & $\begin{array}{l}\mathrm{Ca} \\
\mathrm{ppm}\end{array}$ & $\begin{array}{l}M g \\
p p m\end{array}$ & $\begin{array}{l}\mathrm{Mn} \\
\mathrm{ppm}\end{array}$ & $\begin{array}{l}F e \\
p p m\end{array}$ & $\begin{array}{l}Z n \\
p p m\end{array}$ & $\begin{array}{l}\mathrm{Cu} \\
\mathrm{ppm}\end{array}$ & \\
\hline Apm $20 \%$ & $0-20$ & 0,42 & 3,5 & 2,7 & - & 58,3 & 447 & 48,1 & 12,3 & 673 & 10,0 & 3,3 & 13,2 \\
\hline Ap $80 \%$ & $0-20$ & ,11 & 3,5 & 3,6 & - & 20,0 & 77,3 & 21,1 & 15,1 & 619 & 3,9 & 3,6 & 3,0 \\
\hline$A_{1}$ & $20-42$ & 1,42 & 3,6 & 3,2 & - & 8,0 & 68,2 & 10,1 & 14,9 & 534 & 2,3 & 2,3 & 1,3 \\
\hline$B_{11}$ & $42-90$ & 1,47 & 3,9 & 3,7 & - & 4,3 & 41,2 & 7,8 & 10,5 & 506 & 1,6 & 2,0 & 0,5 \\
\hline$B_{12}$ & $90-100$ & 1,52 & 4,2 & 4,2 & - & 4,9 & 50,0 & 8,7 & 11,5 & 517 & 2,7 & 2,1 & 0,3 \\
\hline $\mathrm{B}_{2} \mathrm{~h}$ & 100-104 & 1,50 & 4,0 & 3,9 & - & 9,9 & 45,9 & 9,0 & 11,1 & 998 & 2,5 & 2,2 & 2,8 \\
\hline $\mathrm{B}_{2} \mathrm{~s}$ dur & $104-110$ & 1,50 & 4,4 & 4,2 & - & 4,3 & 43,9 & 7,8 & 14,1 & 2518 & 3,2 & 2,6 & 1,5 \\
\hline $\mathrm{B}_{2} \mathrm{~s}$ mou & $>110$ & 1,71 & 4,5 & 4,3 & - & 6,1 & 42,7 & 7,1 & 9,2 & 836 & 2,2 & 2,7 & 0,3 \\
\hline
\end{tabular}

eux, l'impact du recyclage biologique par la végétation apparaît clairement. II en va cependant différemment du fer, qui a tendance à s'accumuler dans les horizons $B$, notamment dans les sites de Berganton, Sore et Castillonville - ce qui confirme le fort degré de podzolisation de ces profils.
Si l'on se réfère aux amplitudes de concentration en cuivre citées par Coppenet (1968) ou par Kabata-Pendias et Pendias (1982) pour une large gamme de roches mères ou de sols à la surface du globe, les valeurs obtenues se situent à un niveau exceptionnellement faible. $\mathrm{La}$ 
Tableau X. Analyses de sol, Site de Castillonville.

\begin{tabular}{|c|c|c|c|c|c|c|c|c|c|c|c|c|c|}
\hline \multirow[b]{2}{*}{ Horizon } & \multirow{2}{*}{$\begin{array}{l}\text { Profon- } \\
\text { deur } \\
(\mathrm{cm})\end{array}$} & \multirow{2}{*}{$\begin{array}{l}\text { Den- } \\
\text { sité } \\
\text { app. }\end{array}$} & \multirow{2}{*}{$\begin{array}{l}\mathrm{pH}- \\
\text { eau }\end{array}$} & \multirow{2}{*}{$\begin{array}{l}\mathrm{pH}- \\
\mathrm{KCl}\end{array}$} & \multirow{2}{*}{$\begin{array}{l}N \\
\text { Kjeld. } \\
\text { p. } 1000\end{array}$} & \multicolumn{3}{|c|}{ El. échang. (ac. $\left.\mathrm{NH}_{4}\right)$} & \multicolumn{4}{|c|}{ Eléments totaux (eau régale) } & \multirow{2}{*}{$\begin{array}{l}\text { Mat. } \\
\text { org. } \\
\%\end{array}$} \\
\hline & & & & & & $\begin{array}{l}\text { K } \\
p p m\end{array}$ & $\begin{array}{l}\mathrm{Ca} \\
\mathrm{ppm}\end{array}$ & $\begin{array}{l}\text { Mg } \\
p p m\end{array}$ & $\begin{array}{l}\text { Mn } \\
\text { ppm }\end{array}$ & $\begin{array}{l}F e \\
p p m\end{array}$ & $\begin{array}{l}Z n \\
p p m\end{array}$ & $\begin{array}{l}\mathrm{Cu} \\
\mathrm{ppm}\end{array}$ & \\
\hline & & & & & & & & & & & & & 2 \\
\hline & & & & & & & & & & & & & 27,0 \\
\hline & & & & & & & 8 & & & & & 2 & 1,4 \\
\hline & 37 & $1,3^{*}$ & 4,2 & 3 & & 16 & 73 & 12 & 9 & & & 1,3 & 6,0 \\
\hline & & & 4,3 & & & 10 & 61,2 & 10,1 & 10 & & & 1,9 & 1 \\
\hline & & & & & 0,22 & 11,2 & 128 & 9,6 & 9,2 & 644 & 2,9 & 1,0 & 0,6 \\
\hline
\end{tabular}

*: Selon Righi (1977).

remontée biologique de ce métal et sa forte rétention dans les horizons de surface interviennent donc de manière prépondérante dans la compréhension de sa distribution actuelle le long du profil. Cependant, même dans les horizons de surface (à l'exception de la litière), les concentrations observées se situent nettement en deçà des seuils de carence relatifs aux plantes de grande culture : Duval et Maurice (1970) estiment en effet ce seuil à 7-8 ppm pour les sols acides.

L'intervention déterminante de la végétation dans la "remontée» biologique des éléments-traces est bien illustrée par le Tableau XI, qui fait apparaître par ailleurs que l'intensité de l'accumulation dans l'horizon de surface varie fortement en fonction de l'élément considéré; le fer et, dans une moindre mesure, le cuivre dans les sites de Sore et du Porge s'accumulent préférentiellement car sans doute plus fortement liés à la matière organique.

Tableau XI. Indices de concentration des oligoéléments entre les aiguilles de $2^{\circ}$ année et la litière (Rapport teneur litière/teneur aiguille $2^{\circ}$ année).

\begin{tabular}{lccc}
\hline Elément & Saint-Jean-d'lllac & Le Porge & Sore \\
\hline $\mathrm{Fe}$ & 14,5 & 7,7 & 12,2 \\
$\mathrm{Mn}$ & 4,2 & 1,8 & 1,2 \\
$\mathrm{Zn}$ & 3,1 & 1,3 & 1,1 \\
$\mathrm{Cu}$ & 2,9 & 3,9 & 3,1 \\
\hline
\end{tabular}

\section{Conclusion}

Cette étude à caractère assez systématique, qui visait surtout à mettre en relation la réserve en certains éléments traces de profils caractérisant 5 stations de sols sableux des Landes de Gascogne, bien définis, avec la composition minérale des organes accumulateurs de la strate forestière correspondante, a permis de confirmer ou de mettre en évidence :

- le faible niveau général des réserves totales du substrat en manganèse, zinc, cuivre, facilement explicable par la nature essentiellement quartzeuse de la roche mère;

- bien que l'insuffisance de données disponibles n'ait permis qu'une estimation approximative de l'oligominéralomasse immobilisée dans l'appareil foliaire, il semble cependant que les niveaux des réserves totales du sol soient d'un ordre de grandeur permettant de satisfaire pendant plusieurs décennies les besoins du peuplement;

- le rôle prépondérant de la végétation dans la mobilisation et la redistribution de ces éléments;

- la faiblesse très générale des niveaux de cuivre dans les tissus du Pin maritime (faiblesse jugée il est vrai en faisant référence aux seuils critiques observés chez d'autres espèces atteintes de carence 
bien identifiée en cet élément), qui peut conduire à suspecter a priori une alimentation en cuivre insuffisante chez le Pin maritime dans les sols sableux des Landes de Gascogne, surtout dans les peuplements ayant subi une fertilisation phosphatée. La pauvreté en cet élément du sable quartzeux constituant la roche mère, la relative accumulation de ce métal dans les horizons de surface les plus riches en matière organique, sa très énergique rétention par cette dernière, l'existence de carences en cuivre identifiées sur maïs cultivés dans le même secteur, la nature des malformations observées dans certains peuplements, et surtout la baisse de l'assimilation du cuivre des semis après apport de phosphate constituent autant d'arguments militant en faveur de cette hypothèse. Ils justifient donc un effort de recherche destiné à apprécier la biodisponibilité pour le Pin maritime du cuivre présent dans ce contexte pédologique bien particulier.

\section{Remerciements}

Plusieurs personnes ont fourni, grâce à leurs compétences respectives, une aide précieuse à ce travail. II convient donc de remercier particulièrement : M. C. Juste ' et M. J. Gelpe ${ }^{2}$ pour leur appui; MM. A. Gomez' pour ses conseils en chimie analytique, M. Guedon et M. Sartore ${ }^{2}$ pour la réalisation des récoltes d'échantillons sur le terrain.

\section{Références}

Bonneau M., Gelpe J. \& Illy G. (1972) Résultats de deux essais de fertilisation sur pin maritime

\footnotetext{
' Station d'agronomie, INRA, Domaine de la GrandeFerrade, 33140 Pont-de-La-Maye;

2 Laboratoire de sylviculture et d'écologie, INRA, Domaine de l'Hermitage, Pieroton, 33610 Cestas.
}

adulte dans les Landes. Ann. Sci. For. (Paris) 29, 353-364

Bonneau M., Gelpe J. \& Le Tacon F. (1968) Influence des conditions de nutrition minérale sur le dépérissement du pin maritime dans les Landes de Gascogne. Ann. Sci. For. (Paris) 25, 251-289

Coppenet M. (1968) Les oligo-éléments. Bull. Tech. Inf. $\mathrm{n}^{\circ}$ spécial 23, 595-608

Demounem R. (1979) Essai de définition et de caractérisation de niveaux écophysiologiques dans le massif forestier des Landes de Gascogne Thèse de Doctorat, Univ. de Grenoble

Duval L. \& Maurice J. (1970) Le diagnostic des carences en oligo-éléments au moyen de l'analyse chimique des sols. Ann. Agron. (Paris) 21 (5), 573-586

Gessel S.P., Steinbrenner E.C. \& Miller R.E. (1979) Response of North West forests to elements other than nitrogen. In : Proceedings of Forest Fertilization Conference (Gessel S.P., Kenady R.M. \& Alkinson W.A. eds), Univ. of Washington, College of Forest Resources, Inst. of Forest Resources, Contribution $n^{\circ} 40, \mathrm{pp}$. 140-149

Humphreys F.R. \& Truman R. (1964) Aluminium and the phosphorus requirements of Pinus radiata. Plant Soil 20, 131-134

Juste C. (1965) Contribution à l'étude de la dynamique de l'aluminium dans les sols acides du Sud-Ouest atlantique : application à leur mise en valeur. Thèse de Docteur-Ingénieur, Univ. de Nancy

Kabata-Pendias A. \& Pendias H. (1984) Trace Elements in Soils and Plants. CRC Press

Lemoine B., Gelpe J., Ranger J. \& Nys C. (1986) Biomasse et croissance du pin maritime. Etude de la variabilité dans un peuplement de 16 ans. Ann. Sci. For. (Paris) 43, 67-84

Lemoine B., Ranger J. \& Gelpe J. (1989) Distribution qualitative et quantitative des éléments nutritifs dans un jeune peuplement de pin maritime (Pinus pinaster Ait.) (à paraître)

Loue A. (19816) Les Oligo-éléments en Agriculture. Agri. Nathan, Paris

Messenger A.S. (1975) Climate, time and organisms in relation to podzol development in Michigan sand; II : Relationships between che- 
mical element concentrations in mature tree foliage and upper humic horizons. Soil Sci. Soc. Amer. Proc. 39, 698-702

Penningsfeld F. (1964) Nährstoffmangelerscheinungen bei Baumschulgehölzen. Phosphorsäure 24, 3/4, 199-212

Rahimi A. \& Bussler W. (1974) Kusfermangel bei höheren Pflanzen und sein histochemicher Nachweir. Landwirtsch. Forsch. 27, 101-111

Redlich G.S. (1954) Les problèmes de la culture des sables des Landes. Bull. CETA 15, 149151

Righi D. (1977) Genèse et évolution des podzols et des sols hydromorphes des landes du Médoc. Thèse de Doctorat, Univ. Poitiers

Rodier J. (1954) Analyse de l'Eau, Eaux Naturelles, Eaux Résiduaires, Eau de Mer. Dunod, Paris

Ruiter J.H. (1969) Suspected copper deficiency in radiata Pine. Plant Soil 31, 197-200

Strullu D.G. \& Bonneau M. (1978) Contribution à l'étude des carences en cuivre chez les abiétacées. Can. J. Bot. 56, 20, 2648-2659
Turvey U.D. (1984) Copper deficiency in Pinus radiata planted in a podzol in Victoria, Australia. Plant Soil 77, 73-86

Van Goor C.P. (1963) Bemestingsvoorschrift voor naadlhoutculturen (Fertilization of conifer plantations). Korte Meded. Sticht. BosbProefsta 56, 129-142

Van Goor C.P. (1965) Spurelemente bei der Ernährung von Koniferen, Vortrag Anl. d. Tagung d. Arbeitsgem. Forstdüngung in Tharandt 10, 4-6

Will G.M. (1972) Copper deficiency in radiata pine planted on sand at Mangashai forest. N.Z. J. For. Sci. 2, 2, 277-221

Woods R.V. (1983) Trace element problems induced by heavy nitrogen fertilization of Pinus radiata in South Australia. Commun. Inst. For. Fenn. 116, 178-182

Zöttl H.W. (1985) Waldschäden und Nährelementversorgung. Düsseldorfer Geobot. Kolloq. 2, Düsseldorf, mars 1985, 31-41 